\title{
Study of Wavelet Thresholding Image De-noising Algorithm Based on Improvement Thresholding Function
}

\author{
Qing Zhu, Lei Cui \\ Beijing University of Technology, School of Software Engineering, Beijing, China \\ ccgszq@bjut.edu.cn, special.lei@gmail.com
}

\begin{abstract}
In order to enhance the de-noising performance of wavelet thresholding de-noising algorithm and increase the degree of similarity between the original signal and de-noising signal, this paper proposes a new thresholding function with adjustment factors based on wavelet thresholding de-noising theory introduced by D. L. Donoho and I. M. Johnstone. First continuity and higher derivative of thresholding function can be obtained by setting adjustment factors, second the difference between the value of the original signal and the one quantized is unlimitedly decreased, finally, according to the noisy signal features and the distributional difference of noise energy, the effective thresholding function can be obtained with different adjustment factors. Objective assessment parameters of de-noising image quality are calculated from the experiment of Matlab simulation, which suggests that the impact of de-noising algorithm with the new thresholding function is better than the traditional soft and hard thresholding de-noising algorithms, furthermore, which lays a good foundation on image enhancement, feature extraction and edge detection.
\end{abstract}

Index Terms - wavelet thresholding de-noising, thresholding function, thresholding selection, decomposition level, wavelet basis.

\section{Introduction}

Wavelet thresholding de-noising proposed by D. L. Donoho and I. M. Johnstone[1-3] is widely utilized because of its approximately optimality in the sense of minimum mean square error, high calculation speed and high adaptability. Its theoretical basis is that wavelet transformation possesses a strong capacity of data decorrelation, and the energy of a transformed signal is mainly concentrated in the area of the relatively larger wavelet coefficients of wavelet domain. Instead, the noise energy is concentrated in the wavelet domain where the overall wavelet coefficient amplitude of the noise is less than the one of the effective signal, so the validity of the target wavelet coefficients can be determined according to the size of the amplitude of the wavelet coefficients in order to distinguish signals or noise. Then the coefficients of valid signal are retained via selecting an appropriate threshold value. Instead, the coefficients of the noise signal are reduced to 0 so as to achieve the purpose of de-noising [1-9].

Wavelet thresholding de-noising algorithm is that a noisy signal is decomposed based on a particular wavelet basis and a specific decomposition scale in order to obtain wavelet decomposition coefficients. After compared with threshold value, the coefficients are quantified and reconstructed by thresholding function so as to obtain the optimal estimate of the original signal.

The main factors that influence effect of wavelet thresholding de-noising consist of wavelet basis, decomposition scale, thresholding function and selection of threshold value. The focus of this paper is the selection of thresholding function, that is to propose an improved thresholding function. Wavelet basis, decomposition scale and selection of threshold value are determined based on original research foundation.

\section{Wavelet Thresholding Function And Selection Of Threshold Value}

Major thresholding function

(1) Hard thresholding function

$$
\widehat{w}_{j, k}=\left\{\begin{array}{r}
0,\left|w_{j, k}\right|<\lambda \\
w_{i, k},\left|w_{i, k}\right| \geq \lambda
\end{array}\right.
$$

There exists a discontinuous point under the case of $\mathrm{w}_{\mathrm{j}, \mathrm{k}}=\lambda$ in the function, so additional oscillation is produced and de-noising effect is weaken during the period of image signal processing. But the local features, like the edge of image signal, can be well retained.

(2) Soft thresholding function

$$
\widehat{w}_{j, k}=\left\{\begin{array}{r}
0,\left|w_{j, k}\right|<\lambda \\
\operatorname{sgn}\left(w_{i, k}\right)\left(\left|w_{i, k}\right|-\lambda\right),\left|w_{i, k}\right| \geq \lambda
\end{array}\right.
$$

Under the case of $\left|w_{i, k}\right| \geq \lambda$, there appears a constant deviation $\lambda$ between $\widehat{w}_{\mathrm{j}, \mathrm{k}}$ and $\mathrm{w}_{\mathrm{j}, \mathrm{k}}$, which will lead to expand the deviation between the image signal and the reconstructed signal, thus there appears distortion. Instead, additional oscillation is not produced in the process of image processing due to its excellent continuity and smooth transition. In addition, the derivative of the soft thresholding function is not continuous, and the first derivative, even higher derivative, should be processed in the actual signal processing, so soft thresholding function has certain limitations [10].

(3) Semi-soft thresholding function

$$
\begin{array}{r}
\widehat{\mathrm{w}}_{\mathrm{j}, \mathrm{k}}=\operatorname{sgn}\left(\mathrm{w}_{\mathrm{j}, \mathrm{k}}\right) \\
\frac{\lambda_{2}\left(\left|\mathrm{w}_{\mathrm{j}, \mathrm{k}}\right|-\lambda_{1}\right)}{\lambda_{2}-\lambda_{1}} \mathrm{I}\left(\lambda_{1}<\mathrm{w}_{\mathrm{j}, \mathrm{k}}<\lambda_{2}\right) \\
+\mathrm{w}_{\mathrm{j}, \mathrm{k}} \mathrm{i}\left(\left|\mathrm{w}_{\mathrm{j}, \mathrm{k}}\right|>\lambda_{2}\right) 0<\lambda_{1}<\lambda_{2}
\end{array}
$$

$\mathrm{I}(\mathrm{x})$ is indicative function, $\operatorname{sgn}(\mathrm{O}$ is the sign function.

The function can stably process the image signal and combine the advantages of hard and soft thresholding functions. But it needs to estimate two threshold values, that are $\lambda_{1}$ and $\lambda_{2}$, thus it is not widely applied due to its cumbersome calculation. 
Classical threshold value selection methods

(1) VisuShrink thresholding is derived based on the application of multi-dimensional independent normal variable decision theory. The formula is

$$
\lambda=\sigma \sqrt{2 \log \mathrm{N}}
$$

(2) RigrSure thresholding is derived based on the adaptive thresholding selection of the unbiased likelihood estimation principle. Unbiased likelihood estimation threshold value can be expressed as:

$$
\lambda=\sigma \sqrt{\mathrm{s}_{\alpha}}
$$

(3) HeurSure thresholding is the compromise of RigrSure thresholding and VisuShrink thresholding, and optimal predictive variable thresholding. VisuShrink thresholding is $\lambda_{\mathrm{v}}=\sigma \sqrt{2 \log \mathrm{N}}$ and RigrSure thresholding is $\lambda_{\mathrm{r}}=\sigma \sqrt{\mathrm{s}_{\alpha}}$, $\theta=\frac{\left(\sum_{\mathrm{k}=1}^{\mathrm{N}} \mathrm{w}_{\mathrm{j}, \mathrm{k}}{ }^{2}\right)-\mathrm{N}}{\mathrm{N}}$ and $\mu=\left(\log _{2} \mathrm{~N}\right)^{3 / 2} / \mathrm{N}^{3 / 2}$, then the heuristic threshold value is

$$
\lambda=\left\{\begin{aligned}
\lambda_{v}, & \theta<\mu \\
\min \left(\lambda_{v}, \lambda_{\mathrm{r}}\right), & \theta \geq \mu
\end{aligned}\right.
$$

(4) MiniMaxi thresholding is a fixed thresholding selection method and generate a minimum mean square error extremum. The formula is

$$
\lambda=\left\{\begin{array}{rr}
0, & N \leq 32 \\
\sigma\left(0.396+0.1829 * \log _{2} N\right), & N>32
\end{array}\right.
$$

$\mathrm{N}$ represents the length of the image signal, $\sigma$ represents the noise standard deviation.

\section{Description And Implementation Of The New Thresholding Function}

New thresholding function

$$
\widehat{w}_{j, k}= \begin{cases}\operatorname{sgn}\left(w_{j, k}\right)\left(\left|w_{j, k}\right|-\frac{\alpha \lambda}{\ln \left(e+\beta\left|w_{j, k}\right|\right)}+\frac{\lambda}{2 \mu+1}\right), & \left|w_{j, k}\right| \geq \lambda \\ \frac{1}{(2 \mu+1) \lambda^{2 \mu}} w_{j, k}^{2 \mu+1,} & \left|w_{j, k}\right|<\lambda\end{cases}
$$

or

$$
\widehat{w}_{j, k}=\left\{\begin{array}{ll}
\operatorname{sgn}\left(w_{j, k}\right)\left(\left|w_{j, k}\right|-\frac{\alpha \lambda}{\ln \left(e+\beta\left|w_{j, k}\right|\right)}\right),\left|w_{j, k}\right| \geq \lambda \\
0, & \left|w_{j, k}\right|<\lambda
\end{array}, \mu \rightarrow \infty\right.
$$

$\alpha, \beta$ and $\mu$ is the adjustment factors $(\alpha, \beta, \mu \geq 0), \lambda$ is a threshold value.

In the new thresholding function formula (8), it makes $\widehat{\mathrm{w}}_{\mathrm{j}, \mathrm{k}}$ possess continuity under the case of $\left|\mathrm{w}_{\mathrm{i}, \mathrm{k}}\right|=\lambda$ when

$$
\alpha=1, \beta=0 \text {, that is } \widehat{\mathrm{w}}_{\mathrm{j}, \mathrm{k}}=\left\{\begin{array}{c}
\frac{\lambda}{2 \mu+1}, \mathrm{w}_{\mathrm{j}, \mathrm{k}}=\lambda \\
-\frac{\lambda}{2 \mu+1}, \mathrm{w}_{\mathrm{j}, \mathrm{k}}=-\lambda
\end{array} .\right.
$$

The difference between $\widehat{w}_{\mathrm{j}, \mathrm{k}}$ and $\mathrm{w}_{\mathrm{j}, \mathrm{k}}$ can be infinitely shortened by setting the adjustment factor $\mu$. The thresholding function is high derivable throughout the interval and simply need to estimate only one threshold value $\lambda$. It possesses the advantage of easy calculation.

In the new thresholding function formula (9), the function is approximately similar to hard thresholding function when $\alpha \rightarrow 0$ or $\beta \rightarrow \infty$ and soft thresholding function when $\beta \rightarrow 0, \alpha \rightarrow 1$. The new thresholding function is more flexible due to the adjustment factors. An applicable and appropriate thresholding function is obtained by setting $\alpha, \beta, \mu$.

Description of improved algorithm

(1) Wavelet decomposition of the image signal.

The noisy image signal is decomposed under the case of wavelet basis sym8 and decomposition scale $\mathrm{J}=4$, then the low-frequency coefficients under the case of $J=4$ and highfrequency coefficients $w_{j, k}$ under each decomposition scale are obtained.

(2) High-frequency coefficient thresholding quantization of wavelet decomposition.

High-frequency coefficients $w_{j, k}$ under each decomposition scale obtained in step (1) are quantified with formula (9) and the specific threshold value and the lowfrequency signal is retained. Wavelet high-frequency coefficients $\widehat{w}_{j, k}$ quantified via the threshold function (9) are obtained.

(3) Reconstruction of quantified wavelet coefficients.

The de-noising image signal is reconstructed via wavelet reconstruction with the low-frequency coefficients retained in the Nth layer and the high-frequency coefficients $\widehat{w}_{\mathrm{j}, \mathrm{k}}$ quantified from the first to the Nth layer.

\section{Simulation Experimental Results And Analysis}

Objective assessment methods

The de-noising effect of the image is objectively evaluated with objective quality assessment parameters such as

(1) Mean Squared Error

$$
\mathrm{MSE}=\frac{1}{\mathrm{~N}} \sum_{\mathrm{i}=1}^{\mathrm{N}}[\mathrm{X}(\mathrm{i})-\widehat{\mathrm{X}}(\mathrm{i})]^{2}
$$

(2) Signal Noise Ration

$$
\mathrm{SNR}=10 \log _{10} \frac{\sum_{\mathrm{i}=1}^{\mathrm{N}} \mathrm{x}^{2}(\mathrm{i}) / \mathrm{N}}{\mathrm{MSE}}(\mathrm{dB})
$$

(3) Peak Signal to Noise Ration

$$
\text { PSNR }=10 \log _{10} \frac{255^{2} / \mathrm{N}}{\mathrm{MSE}}
$$

(4) Root-Mean Square Error

$$
\operatorname{RMSE}=\left(\frac{1}{\mathrm{~N}} \sum_{\mathrm{i}=1}^{\mathrm{N}}[\mathrm{X}(\mathrm{i})-\widehat{\mathrm{X}}(\mathrm{i})]^{2}\right)^{\frac{1}{2}}
$$

$\mathrm{X}(\mathrm{i})$ is the original image signal and $\widehat{\mathrm{X}}(\mathrm{i})$ is the quantified image signal, $\mathrm{N}$ is the length of image signal.

Experimental results and analysis

(1) Experiment one: comparison of the de-noising performance of the different thresholding functions.

In this experiment, the crack images, lena.jpg $(512 * 512)$ and crack.jpg $(512 * 512)$, are considered as research objects, and 
then are relatively processed and quantified via hard-soft thresholding functions and the new thresholding function under the case of wavelet basis sym8, decomposition scale $\mathrm{J}=4$ and Gaussian noise $\sigma=0.01$.

TABLE I Contrast of Objective Assessment Parameters of De-noising Image For Lena

\begin{tabular}{|l|l|l|l|}
\hline $\begin{array}{c}\text { Evaluation } \\
\text { parameters }\end{array}$ & $\begin{array}{l}\text { soft } \\
\text { threshold } \\
\text { function }\end{array}$ & $\begin{array}{l}\text { hard } \\
\text { threshold } \\
\text { function }\end{array}$ & $\begin{array}{l}\text { new function } \\
(\boldsymbol{\mu} \rightarrow \infty, \boldsymbol{\alpha}=\mathbf{0 . 5}, \boldsymbol{\beta}=\mathbf{1 0})\end{array}$ \\
\hline SNR & 74.3434 & 75.8283 & 76.0718 \\
RMSE & 0.0431 & 0.0363 & 0.0353 \\
MSE & 0.0019 & 0.0013 & 0.0012 \\
PSNR & 75.4477 & 76.9425 & 77.1760 \\
\hline
\end{tabular}

TABLE II Contrast of Objective Assessment Parameters of De-noising Image For Crack

\begin{tabular}{|l|l|l|l|}
\hline $\begin{array}{c}\text { Evaluation } \\
\text { parameters }\end{array}$ & $\begin{array}{c}\text { soft threshold } \\
\text { function }\end{array}$ & $\begin{array}{c}\text { hard threshold } \\
\text { function }\end{array}$ & $\begin{array}{c}\text { new function } \\
(\boldsymbol{\mu} \rightarrow \infty, \\
\boldsymbol{\alpha}=\mathbf{0 . 5}, \\
\boldsymbol{\beta}=\mathbf{1 0})\end{array}$ \\
\hline SNR & 76.9800 & 77.2100 & 77.3297 \\
RMSE & 0.0405 & 0.0394 & 0.0389 \\
MSE & 0.0016 & 0.0016 & 0.0015 \\
PSNR & 75.9823 & 76.2124 & 76.3321 \\
\hline
\end{tabular}

From Table 1 and Table 2, compared with objective assessment parameter values of soft-hard thresholding functions, the new thresholding functions' are more optimal when $\mu \rightarrow \infty, \alpha=0.5, \beta=10$. The advantages mainly embody in improvement of image SNR and similarity, error reduction between the original image signal and de-noising image signal, low distortion rate.

signal and de-noising image signal, low distortion rate.

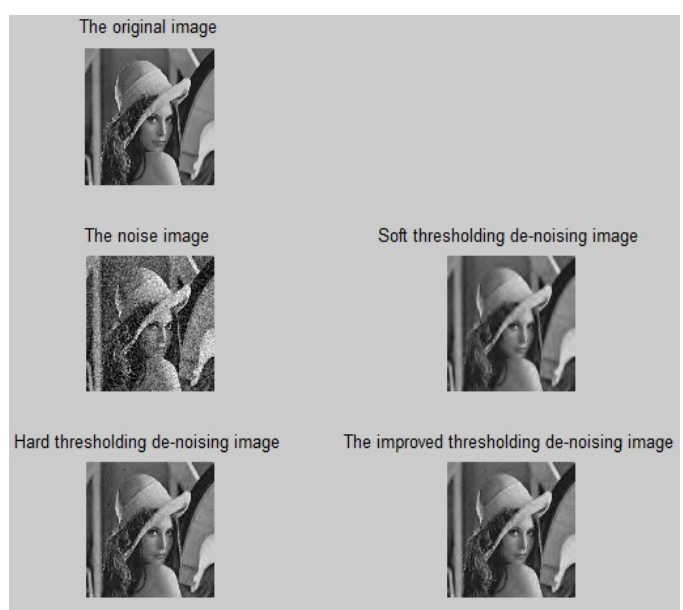

Figure 1. Image de-noising simulation effect diagram of lena

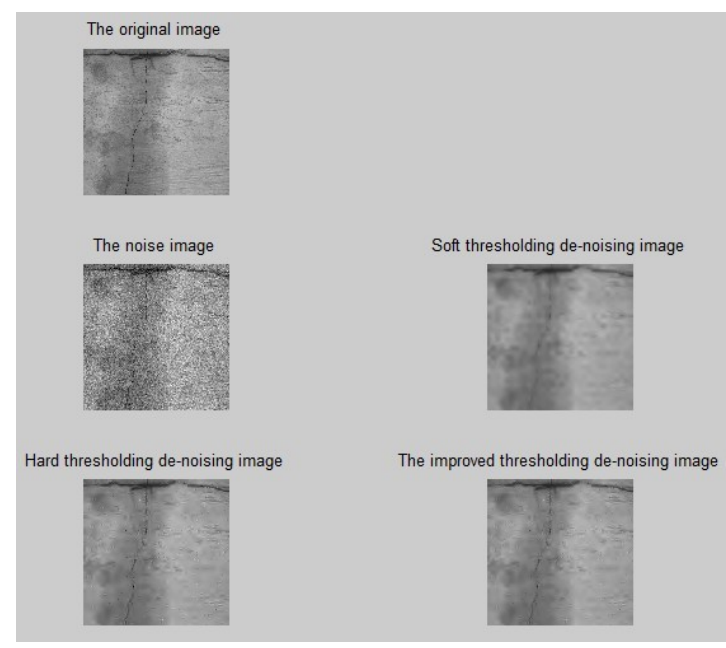

Figure 2. Image de-noising simulation effect diagram of crack

From the Figure 1 and Figure 2, edges of the soft thresholding de-noising image appear blurred, smooth transition of image details and poor reconstruction accuracy, which result in distortion. The de-noising image via the method of hard thresholding exists noise points (pseudo-Gibbs phenomenon). Instead, the de-noising effect of the new method, from the subjective point of view, possesses lowdistortion, the less number of noise points, smooth and natural transition of image details, high reconstruction accuracy and high SNR so that the similarity between the original image signal and the de-noising image signal is improved.

Experiment one describes that the noise images with different characteristics are quantified and processed via different thresholding functions under the case of Gaussian noise $\sigma=0.01$. Objective evaluation parameter values and subjective evaluation effect have proved that the new thresholding function possesses certain advantages in the field of wavelet thresholding image de-noising.

(2) Experiment two: performance comparison of the new thresholding function with different adaptive thresholding.

In this experiment, the image, lena.jpg $(512 * 512)$, is considered as research object under the case of wavelet basis sym8, decomposition scale $\mathrm{J}=4$ and relatively Gaussian noise $\sigma=0.005$ and $\sigma=0.015$. The new thresholding function is in combination with different threshold values, such as VisuShrink, RigrSure, HeurSure and Minimaxi so that different groups are constructed. The difference of each group's image quality objective assessment parameter values are compared with other groups. 
TABLE III Contrast of Objective Assessment Parameters

\begin{tabular}{|c|c|c|c|c|}
\hline & \multicolumn{4}{|c|}{$\sigma=0.005$} \\
\hline \multirow[b]{2}{*}{ RigrSure } & SNR & RMSE & MSE & $\begin{array}{l}\text { PSNR } \\
\end{array}$ \\
\hline & 72.1282 & 0.0556 & 0.0031 & 73.2324 \\
\hline HeurSure & 69.6907 & 0.0736 & 0.0054 & 70.7950 \\
\hline VisuShrink & 69.6907 & 0.0736 & 0.0054 & 70.7950 \\
\hline Minimaxi & 69.7600 & 0.0730 & 0.0053 & 70.8643 \\
\hline \multirow[t]{3}{*}{ Exp one } & 77.8431 & 0.0288 & $8.2860 \mathrm{e}-004$ & 78.9474 \\
\hline & \multicolumn{4}{|c|}{$\sigma=0.015$} \\
\hline & SNR & RMSE & MSE & PSNR \\
\hline RigrSure & 72.0585 & 0.0560 & 0.0031 & 73.1627 \\
\hline HeurSure & 69.6451 & 0.0740 & 0.0055 & 70.7494 \\
\hline VisuShrink & 69.6451 & 0.0740 & 0.0055 & 70.7494 \\
\hline Minimaxi & 69.7130 & 0.0734 & 0.0054 & 70.8172 \\
\hline Exp one & 74.9766 & 0.0400 & 0.0016 & 76.0808 \\
\hline
\end{tabular}

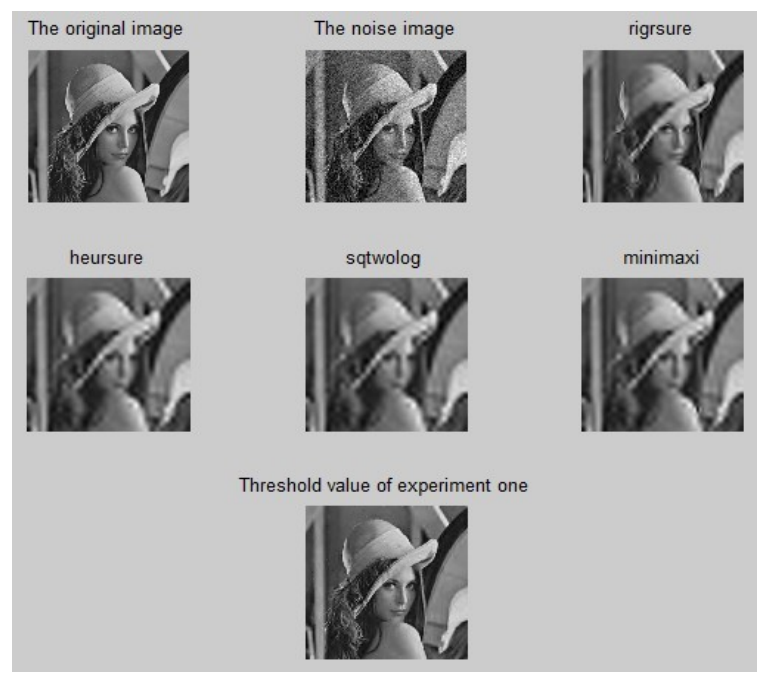

Figure 3. $\sigma=0.005$ De-noising effect diagram

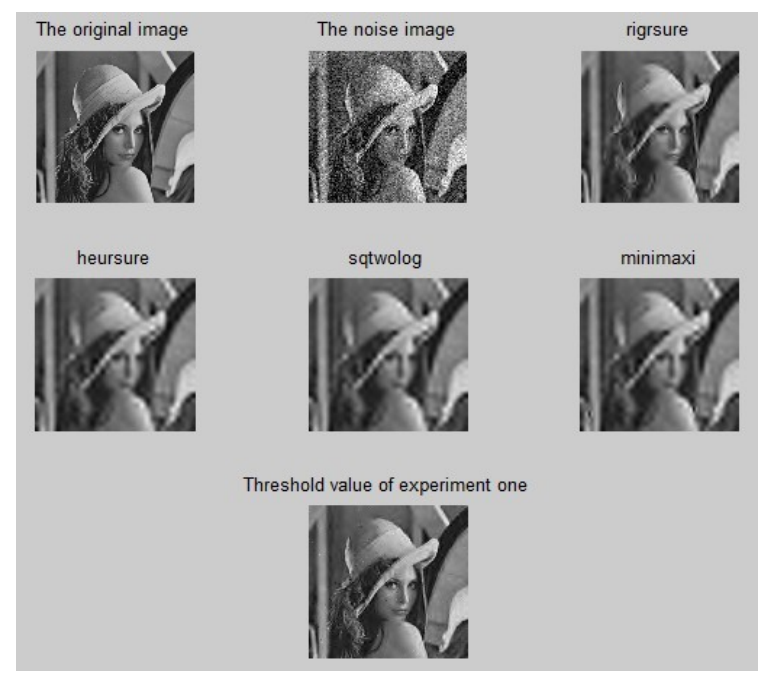

Figure 4. $\sigma=0.015$ De-noising effect diagram
The de-noising image quality objective evaluation parameters of the combination of the new thresholding function and RigrSure is superior to parameters of the combinations of the new thresholding function with HeurSure, VisuShrink or Minimaxi, instead, is inferior to experiment one. From a subjective point of view, the de-noising quality of the experiment one is optimal.

Experiment two proves that in the wavelet thresholding de-noising methods, the selection of the thresholding function and threshold values in some extent influence the de-noising quality of images. Furthermore, the selection is determined mainly based on image features and energy distribution of noise.

\section{Conclusion}

There is a constant error between the actual value and the value of wavelet high-frequency coefficient quantified by soft thresholding function, which leads to influence the similarity between the original image signal and the reconstructed image signal. Hard thresholding function exists breakpoints and discontinuous. While the new thresholding function in some extent makes improvements based on the soft-hard thresholding functions. The de-noising image quality objective assessment parameter values calculated from the MATLAB simulation experiments indicate that the new thresholding function possesses the following advantages

1) Continuity and high-order derivative; the difference between the original signal value and the de-noising quantified one can be infinitely shorten by setting the adjustment factors, which can make up the insufficiency of the existence of constant deviation $\lambda$.

2) The disadvantage of cumbersome calculation of the semi-soft thresholding function is overcome.

3) The appropriate and adaptive thresholding function is obtained by flexibly setting the adjustment factors according to the noise image signals' features and distribution difference of noise energy in order to lay a solid foundation for image enhancement, feature extraction and edge detection.

\section{Acknowledgment}

I would like to extend my sincere gratitude to my mentor, Zhu Qing, for her instructive advice and useful suggestions on my paper. More importantly, she could work and do experiments with me, guide me how to do them and help me avoid detours. I am deeply grateful of her help in the completion of this paper.

\section{References}

[1] DONOHO D L. De-noising by soft-thresholding[J]. IEEE Transformation Theory, 1995, 41(3): 613-627.

[2] DONOHO D L, JOHNSTONE I M. Neo-classical minimax theorems, thresholding and adaptive function estimation[J]. Bernoulli, 1996, 2(1): 39-62.

[3] DONOHO D L, JOHNSTONE I M. Adapting to Unknown Smoothness via wavelet shrinkage[J]. Journal of American Statistical Association, 1995, 90: 1200-1224. 
[4] BRUCE A T, JEFFREY J R. Wavelet-based color image de-noising[C]. IEEE International Conference on Image Processing Proceedings, 2000, (2): 804 807.

[5] GHAZEL M, FREEMAN G H, VRASCAY E R. Fractal-wavelet image de-noising revisited[J]. IEEE Transformation Image Processing, 2006, 15(9): 2669-2675.

[6] CHANG S G, YU B, VETTERLI M. Adaptive wavelet thresholding for image de-noising and compression[J]. IEEE Transformation Image Processing, 2000, 9(9): 1532-1546.

[7] FU Wei, XU Shan-chuan. Improved Algorithm for Threshold De-Noising in Wavelet Transform Domain[J]. Chinese Journal of SENSORS AND ACTUATORS, 2006, 19(2): 534-536, 540.
[8] ZHANG Lian, QIN Hua-feng, YU Cheng-bo. Research of denoising method based on wavelet threshold[J]. Journal of Computer Engineering and Applications, 2008, 44(9): 173-174, 199.

[9] Gonzalez R C,Woods R E. Digital Image Processing, Third Edition [M]. Beijing: Electronic Industry PRESS, 2011. 6.

[10] SUN Yan-kui. Analysis and application of wavelet[M]. Beijing: China Machine PRESS, 2011.6.

[11] HU Chang-hua, LI Guo-hua, LIU Tao, etc. System analysis and design based on MATLAB 6.x [M]. Xi'an: xidian university press, 2004.

[12] ZHOU Wei. Wavelet analysis and application based on MATLAB [M]. Xi'an: xidian university press, 2010. 